\title{
GEANT4 Tuning For pCT Development
}

\author{
Olga Yevseyeva ${ }^{\mathrm{a}}$, Joaquim T. de Assis ${ }^{\mathrm{a}}$, Ivan Evseev ${ }^{\mathrm{b}}$, Hugo R. Schelin ${ }^{\mathrm{b}}$, \\ Sergei A. Paschuk ${ }^{\mathrm{b}}$, Edney Milhoretto ${ }^{\mathrm{b}}$, João A. P. Setti ${ }^{\mathrm{b}}$, Katherin S. \\ Díaz $^{\mathrm{c}}$, Joel M. Hormaza ${ }^{\mathrm{d}}$, Ricardo T. Lopes ${ }^{\mathrm{e}}$ \\ ${ }^{a}$ Instituto Politécnico da Universidade do Estado do Rio de Janeiro, Rua Alberto Rangel, \\ $s / n, 28630-050$ Nova Friburgo, RJ, Brasil \\ ${ }^{b}$ Universidade Tecnológica Federal do Paraná, Av. Sete de Setembro 3165, 80230-901, Curitiba, PR, \\ Brasil \\ ${ }^{c}$ Centro de Aplicaciones Tecnologicas y Desarrollo Nuclear, Havana, Cuba \\ ${ }^{d}$ Instituto de Biociências de Botucatu da UNESP, Distrito de Rubião Jr. s/n ${ }^{\circ}, 18618-000$, Botucatu, SP, \\ Brasil \\ ${ }^{e}$ Laboratório de Instrumentação Nuclear COPPE, UFRJ, Av. Horácio Macedo 2030, 21941-914 Rio de \\ Janeiro, RJ, Brasil
}

\begin{abstract}
Proton beams in medical applications deal with relatively thick targets like the human head or trunk. Thus, the fidelity of proton computed tomography (pCT) simulations as a tool for proton therapy planning depends in the general case on the accuracy of results obtained for the proton interaction with thick absorbers. GEANT4 simulations of proton energy spectra after passing thick absorbers do not agree well with existing experimental data, as showed previously. Moreover, the spectra simulated for the Bethe-Bloch domain showed an unexpected sensitivity to the choice of low-energy electromagnetic models during the code execution. These observations were done with the GEANT4 version 8.2 during our simulations for pCT. This work describes in more details the simulations of the proton passage through aluminum absorbers with varied thickness. The simulations were done by modifying only the geometry in the Hadrontherapy Example, and for all available choices of the Electromagnetic Physics Models. As the most probable reasons for these effects is some specific feature in the code, or some specific implicit parameters in the GEANT4 manual, we continued our study with version 9.2 of the code. Some improvements in comparison with our previous results were obtained. The simulations were performed considering further applications for pCT development.
\end{abstract}

Keywords: Proton computed tomography (pCT), Monte Carlo simulations, GEANT4 code PACS: $82.20 . \mathrm{Wt} ;$ 87.10.Rt; 87.57.Q.

The interest in proton computed tomography (pCT) development is now reopened with the spread of the proton beam treatment [1]. The idea is to use the same medical proton therapy beam for diagnosis with pCT, i.e. for tumor localization and data acquisition for further irradiation planning. Potentially it can improve the quality of proton therapy and decrease the dose delivered to patients. The Monte Carlo simulations have a successful history in varied fields of study and could also be a helpful instrument in the case of pCT. 


\section{MONTE CARLO SIMULATIONS}

By now, the passage of charged particles through matter is studied well enough and some popular Monte Carlo packages could be used to study pCT development. The modern approach to pCT technology involves High Energy Physics (HEP) solutions. Therefore, the GEANT4 Simulation Toolkit [2], which was initially designed to support the development of such HEP system, is very useful for pCT modeling as well [3]. It should be stressed out that here we are concerned with the code ability to support the pCT system development, and not yet for the medical applications.

However, the question is how realistic are the GEANT4 simulations in the pCT case? The pCT deals specifically with relatively thick targets, like the human head or trunk. Our initial comparative simulations [4], [5], [6] with GEANT4 and TRIM from the SRIM2008 package [7], [8] have shown that these two popular Monte Carlo codes usually generate somewhat different proton spectra. In the case of thin absorbers, the difference could be ignored because it is less than could be detected experimentally. For thick absorbers, however, the difference is large enough. Thus, the experimental data could be used for the validation of the code mentioned above. Therefore, from the physics point of view, the question can be reformulated to "How realistic are the GEANT4 simulations for the absorber thicknesses compared with the full ranges of protons [9] in the correspondent matter?" Within the pCT problem, this question is obviously limited to the initial proton energies - equal or below $250 \mathrm{MeV}$ - the maximum proton energy of a typical medical accelerator [10]. On the other hand, the pCT is only possible if the proton after traversing the patient body still has a few $\mathrm{MeV}$ to be detected. Thus, for pCT development only the so-called "Bethe-Bloch domain" of proton energies [9], [11], [12] is important.

Within the Bethe-Bloch domain, the question is almost energy and material independent: the energy-range relations are practically identical if the reduced units for the energy and passed thickness are used [5], [13]. That is why we could take the $19,68 \mathrm{MeV}$ proton data from [14] as the experimental reference without losing the validity of the presented analysis.

It should be stressed out that the original validation of GEANT4 code was done against NIST PSTAR data for the Stopping Power (SP) [15]. In this work, we will focus only in some improvements in simulation results that were achieved in comparison with our previous results.

All simulations are based on Hadrontherapy Advanced Example (distributed with Geant4) with adjusting of some parameters. The simulations were performed with GEANT4 (version 9.2) using the standard electromagnetic pack [16], and with the Low Energy Extension pack [17] using ICRU49 and Ziegler85 models (Ziegler77 and Ziegler2000 models also were tested).

As in the standard configuration, the pack applies the Bethe-Bloch formalism for proton energies down to $2 \mathrm{MeV}$. The models are responsible for the SP parameterization below this boundary energy. Thus, as the simulated energy spectra are from the Bethe-Bloch domain, the differences in the results are somewhat unexpected 


\section{RESULTS AND CONCLUSIONS}

The Figure 1 shows the experimental data in comparison with the results of our previous and actual simulations.

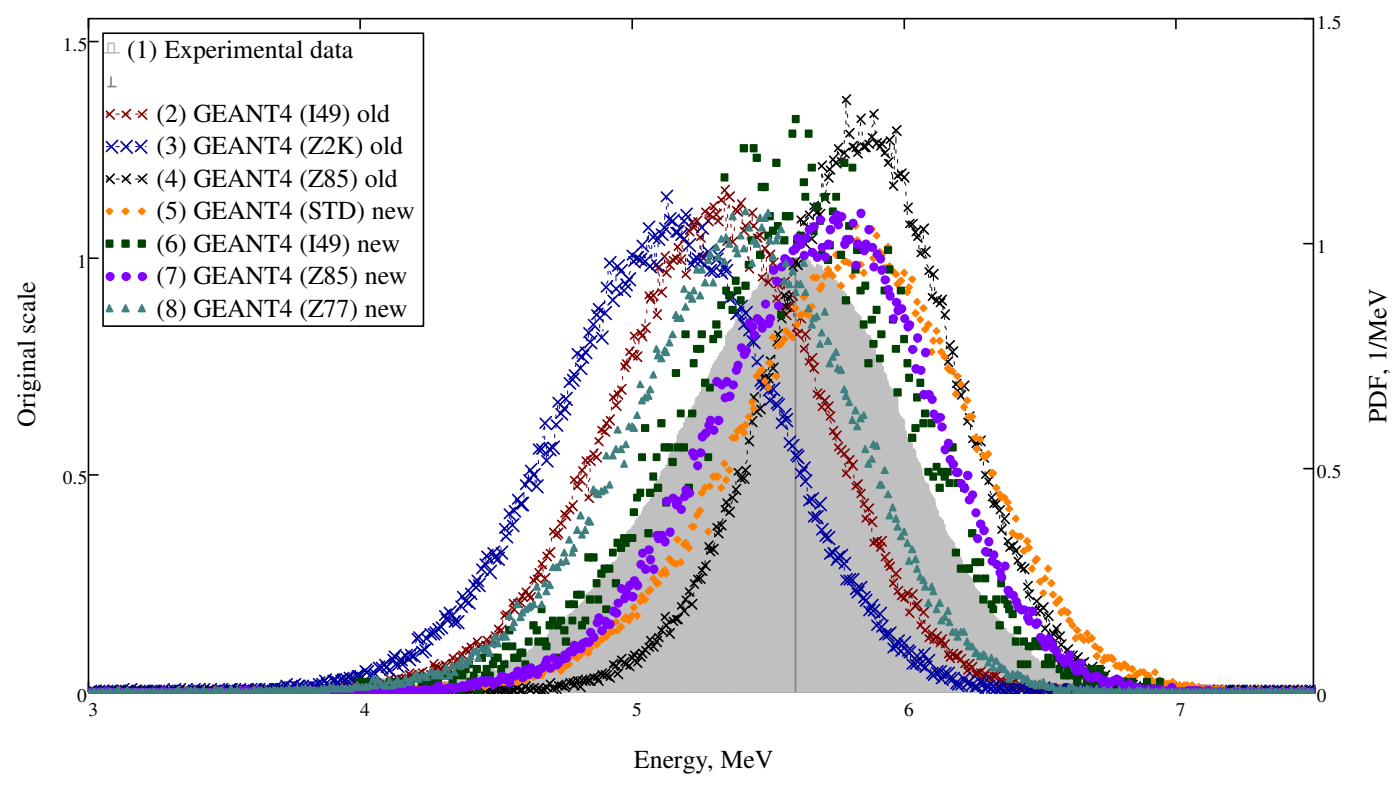

(a) $1,841 \mathrm{~mm}$

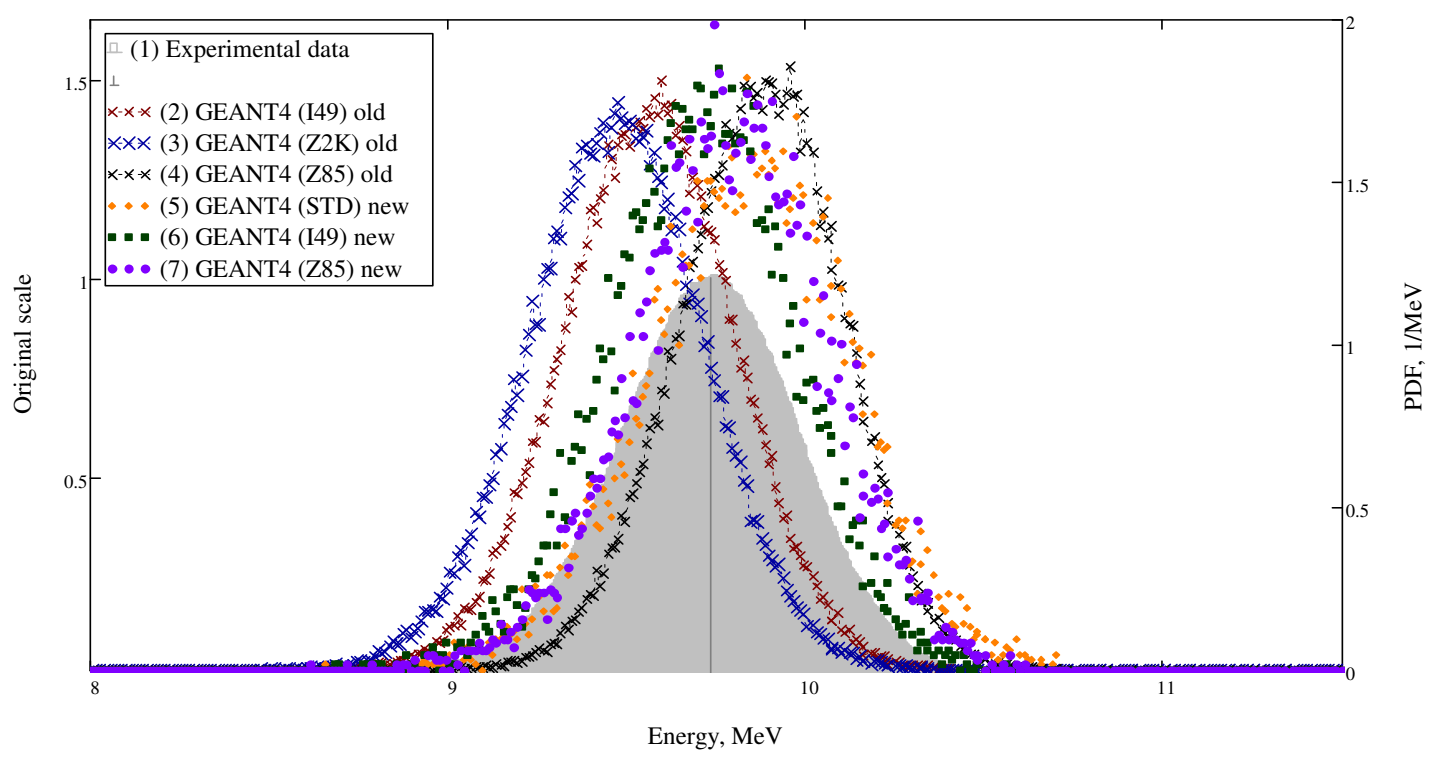

(b) $1,475 \mathrm{~mm}$ 


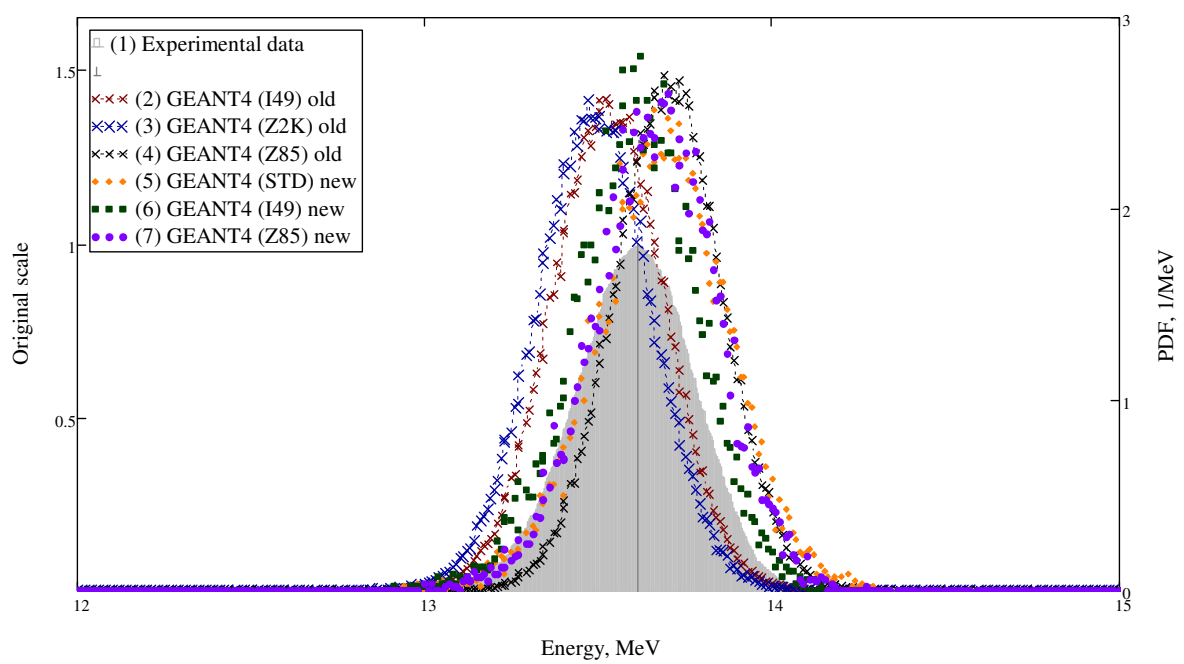

(c) $0,911 \mathrm{~mm}$

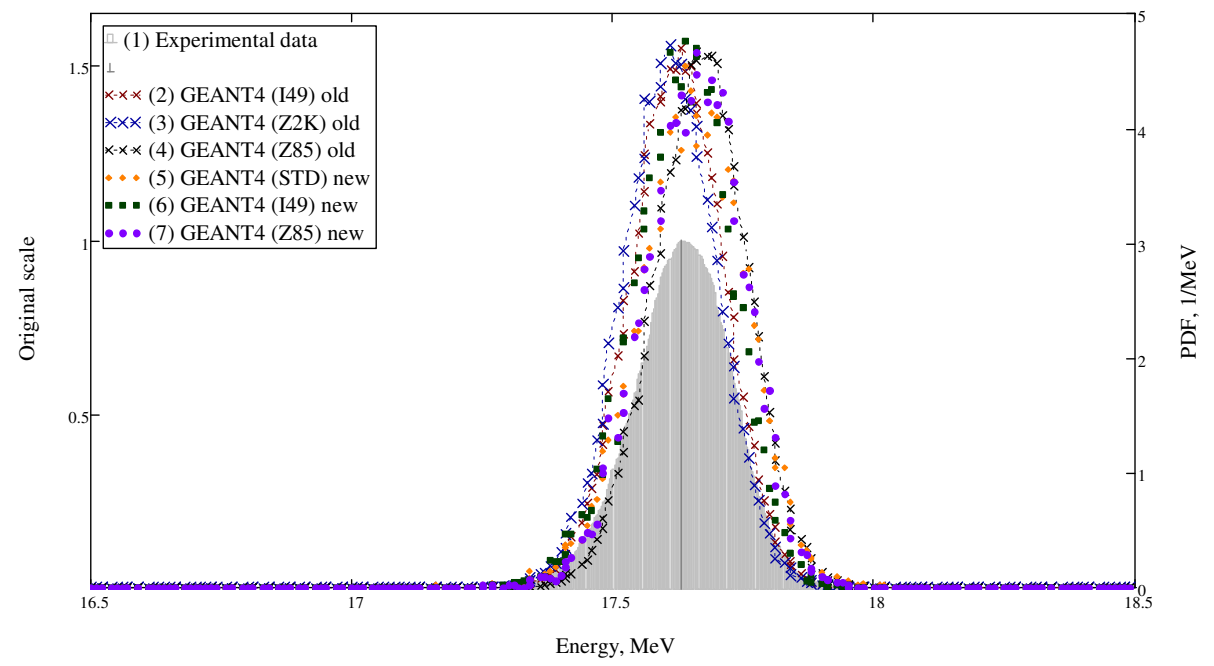

(d) $0,367 \mathrm{~mm}$

FIGURE 1. Final 19.68 MeV proton Energy spectra after Al layer, where (1) - experimental data; GEANT4 simulation results: (2) - 8.2 ICRU49 model, (3) - 8.2 ZIEGLER2000 model, (4) - 8.2 ZIEGLER85 model, (5) - 9.2 default configuration, (6) - 9.2 ICRU49 model, (7) - 9.2 ZIEGLER85 model, (8) - 9.2 ZIEGLER77 model.

The most significant difference in the simulation results was obtained for the thickest Al layer. As could be seen, the results of the actual simulations are in better coincidence with experimental data and with each other, while for the earlier simulations almost the $1 \mathrm{MeV}$ difference was detected. Between the actual simulations the ICRU49 and Ziegler85 models produced better results in comparison with experimental data for this case. This observation stands for all Al layers. It could be 
seen that with the decreasing of the thickness the disagreement between different simulation models and experimental data also decrease.

It was detected that the Low Energy Extension Pack inclusion at the GEANT4 code affects all simulated spectra at the Bethe-Bloch domain. This is important for the pCT development at low energies.

Even the results of our simulations proved to be reliable, the comparison with few experimental spectrums is not sufficient to make a definitive choice for the most adequate way to use GEANT4 for pCT simulations. Thus, we are planning to continue this work involving other experimental data.

\section{ACKNOWLEDGMENTS}

The authors are greatly thankful to Dr. Reinhard Schulte from LLUMC (USA) for useful discussions. The work was supported by "Fundação Araucária" (Paraná State, Brazil), FAPERJ, CAPES and CNPq.

\section{REFERENCES}

1. L. Jonson, B. Keeney, G. Ross, et al., Nucl. Instr. Meth. A514, 215-223 (2003)

2. S. Agostinelli, J. Allison, K. Amako, et al., Nucl. Instr. Meth. A506, 250-303 (2003)

3. I. Evseev, M. C. L. Klock, S. A. Paschuk, et al., Braz. J. Phys. 34, 804-807 (2004)

4. J. T. Assis, O. Yevseyeva, I. Evseev, et al., X-Ray Spectrom. 34, 481-492 (2005).

5. I. Evseev, T. Assis, O. Yevseyeva, et al., "The density measurements in pCT imaging”, In: Medical Imaging 2005: Physics of Medical Imaging. Edited by Flynn, Michael J. Proc. SPIE 5745, 764-774 (2005).

6. I. Evseev, T. Assis, O. Yevseyeva, et al., "Comparison of some popular Monte Carlo Solutions for proton transportation within pCT problem”, In: Proceedings of the 2007 International Nuclear Atlantic Conference - INAC2007, Santos-SP, Brazil, September 30 to October 5, 2007. ABEN E05 1826 (2007).

7. J. F. Ziegler, J. P. Biersack and U. Littmark, The Stopping and Range of Ions in Solids, Pergamon Press, 1985.

8. J. F. Ziegler, Nucl. Instr. Meth. B219-220, 1027-1036 (2004)

9 ICRU Report 49: Stopping Powers and Ranges for Protons and Alpha Particles, International Commission on Radiation Units and Measurements., 1993

10 G. Coutrakon and J. M. Slater, "Design Considerations for Medical Proton Accelerators”, in Proc. 1999 Particle Accelerator Conference, New York, 1999, pp. 11-15.

11 J. F. Ziegler, J. Appl. Phys. 85, 1249-1272 (1999)

12 V.S. Remizovich, D. B. Rogozkin and M. I. Ryazanov, (in Russian) Fizika Elementarnykh Chastits $i$ Atomnogo Yadra 17, No.5, .929-981 (1986).

13 I. Evseev, T. Assis, O. Yevseyeva, et al., Braz. J. Phys. 35, No.3B, 747-750 (2005)

14. C. Tschalär and H. D. Maccabee, Phys. Rev. B1, 2863-2869 (1970).

15 K. Amako, S. Guatelli, V. N. Ivanchenko, et al., IEEE Trans. Nucl. Sci. 52, 910-918 (2005)

16 V. N. Ivanchenko, M. Maire and L. Urban, "Geant4 standard electromagnetic package for HEP applications”, in Proc. Conf. Rec. 2004 IEEE Nucl. Sci. Symp., Rome, Italy, 2004, Paper code: N33179.

17 S. Chauvie, G. Depaola, V. Ivanchenko, et al., "Geant4 low energy electromagnetic physics" In: Proc. Computing in High Energy and Nuclear Physics 2001, Science Press, Beijing, China, 337340. 
Copyright of AIP Conference Proceedings is the property of American Institute of Physics and its content may not be copied or emailed to multiple sites or posted to a listserv without the copyright holder's express written permission. However, users may print, download, or email articles for individual use. 